\title{
HIGHLIGHTS
}

IMAGING

\section{Novel MRI technique distinguishes between glioma and radiation necrosis}

Current imaging techniques are unable to distinguish tumor recurrence from radiation necrosis, which represents a major challenge in neuro-oncology. Furthermore, radiation injury caused by newer antitumor therapies has increased, prompting an urgent need to distinguish these processes. In a study published in Nature Medicine, Jinyuan Zhou et al. have developed a new MRI technique called amide proton transfer (APT) to address this issue, which could represent the first noninvasive technology for the clinic. "We have successfully demonstrated the ability for ATP imaging to distinguish between viable tumors and radiation necrosis in preclinical models," explains Zhou.

The researchers generated rat models that represented radiation necrosis and brain tumors, and showed that current standard MRI techniques could not distinguish between these different pathological processes. Using the APT
MRI approach in various glioma models and models of radiation-induced necrosis, a stark contrast in signal intensity between the different models was demonstrated. Viable glioma displayed a significantly hyperintensive profile whereas a hypointensive-isointensive profile was observed for models of radiation necrosis.

Zhou concludes, "ATP imaging is a totally noninvasive molecular MRI technique that can easily be translated to the clinic using standard MRI platforms." Zhou's team plan to study whether the APT signal-as a specific imaging biomarker-is reliable for assessing brain tumor response to therapy in the clinical setting.

Lisa Hutchinson

Original article Zhou, J. et al. Differentiation between glioma and radiation necrosis using molecular magnetic resonance imaging of endogenous proteins and peptides. Nat. Med.17, 130-134 (2011) 